PROCEEDINGS OF THE

AMERICAN MATHEMATICAL SOCIETY

Volume 133, Number 4, Pages 1007-1012

S 0002-9939(04)07631-2

Article electronically published on October 18, 2004

\title{
POLYNOMIAL DETECTION OF MATRIX SUBALGEBRAS
}

\author{
DANIEL BIRMAJER
}

(Communicated by Martin Lorenz)

\begin{abstract}
The double Capelli polynomial of total degree $2 t$ is

$$
\sum\left\{(\operatorname{sg} \sigma \tau) x_{\sigma(1)} y_{\tau(1)} x_{\sigma(2)} y_{\tau(2)} \cdots x_{\sigma(t)} y_{\tau(t)} \mid \sigma, \tau \in S_{t}\right\} \text {. }
$$

It was proved by Giambruno-Sehgal and Chang that the double Capelli polynomial of total degree $4 n$ is a polynomial identity for $M_{n}(F)$. (Here, $F$ is a field and $M_{n}(F)$ is the algebra of $n \times n$ matrices over $F$.) Using a strengthened version of this result obtained by Domokos, we show that the double Capelli polynomial of total degree $4 n-2$ is a polynomial identity for any proper $F$ subalgebra of $M_{n}(F)$. Subsequently, we present a similar result for nonsplit inequivalent extensions of full matrix algebras.
\end{abstract}

\section{INTRODUCTION}

The double Capelli polynomial of total degree $2 t$ is

$$
\sum\left\{(\operatorname{sg} \sigma \tau) x_{\sigma(1)} y_{\tau(1)} x_{\sigma(2)} y_{\tau(2)} \cdots x_{\sigma(t)} y_{\tau(t)} \mid \sigma, \tau \in S_{t}\right\} .
$$

In this paper we show that the double Capelli polynomial of degree $4 n-2$ is a polynomial identity for any proper subalgebra of $M_{n}(F)$. Subsequently, we present a polynomial test for nonsplit inequivalent extensions of full matrix algebras.

To begin, let $F$ be a field, $M_{n}(F)$ the algebra of $n \times n$ matrices over $F$, and $F\{X\}=F\left\{x_{1}, x_{2}, \ldots\right\}$ the free associative algebra over $F$ in countably many variables. Sometimes we will use other variables $x, y, z, x_{i}, y_{i}$ for simplicity of notation. A nonzero polynomial $f\left(x_{1}, \ldots, x_{m}\right) \in F\{X\}$ is a polynomial identity for an $F$-algebra $R$ if $f\left(r_{1}, \ldots, r_{m}\right)=0$ for all $r_{1}, \ldots, r_{m} \in R$. A $T$-ideal is an ideal of $F\{X\}$ that is closed under endomorphisms of $F\{X\}$. If $f_{1}, \ldots, f_{t}$ are polynomial identities for $R$, so is every polynomial $f$ in the $T$-ideal generated by $f_{1}, \ldots, f_{t}$. In this case we say that the identity $f=0$ in $R$ is a consequence of the identities $f_{i}=0$, for $1 \leq i \leq t$.

The standard polynomial of degree $t$ is

$$
s_{t}\left(x_{1}, \ldots, x_{t}\right)=\sum_{\sigma \in S_{t}}(\operatorname{sg} \sigma) x_{\sigma(1)} x_{\sigma(2)} \cdots x_{\sigma(t)},
$$

Received by the editors November 13, 2003 and, in revised form, December 22, 2003.

2000 Mathematics Subject Classification. Primary 15A24, 15A99, 16 R99.

Key words and phrases. Polynomial identity, polynomial test, matrix subalgebra, double Capelli polynomial. 
where $S_{t}$ is the symmetric group on $\{1, \ldots, t\}$ and $(\operatorname{sg} \sigma)$ is the sign of the permutation $\sigma \in S_{t}$. The standard polynomial $s_{t}$ is homogeneous of degree $t$, multilinear and alternating.

The Amitsur-Levitzki theorem asserts that $M_{n}(F)$ satisfies any standard polynomial of degree $2 n$ or higher. Moreover, if $M_{n}(F)$ satisfies a polynomial of degree $2 n$, then the polynomial is a scalar multiple of $s_{2 n}$ (cf. [1]). The Capelli polynomials are

$$
c_{2 t-1}\left(x_{1}, \ldots, x_{t}, y_{1}, \ldots, y_{t-1}\right)=\sum_{\sigma \in S_{t}}(\operatorname{sg} \sigma) x_{\sigma(1)} y_{1} x_{\sigma(2)} y_{2} \cdots x_{\sigma(t-1)} y_{t-1} x_{\sigma(t)}
$$

and

$$
c_{2 t}\left(x_{1}, \ldots, x_{t}, y_{1}, \ldots, y_{t}\right)=c_{2 t-1}\left(x_{1}, \ldots, x_{t}, y_{1}, \ldots, y_{t-1}\right) y_{t} .
$$

These polynomials were introduced by Razmyslov in [9]. The polynomials $c_{2 t-1}$ and $c_{2 t}$ are multilinear and alternating as a function of $x_{1}, \ldots, x_{t}$. It is clear by a dimension argument that $c_{2 n^{2}}$ is a PI for any proper $F$-subalgebra of $M_{n}(F)$. On the other hand, $c_{2 n^{2}}$ is not a PI for $M_{n}(F)$. To see this, evaluate

$c_{2 n^{2}}\left(x_{1}, \ldots, x_{n^{2}}, y_{1}, \ldots y_{n^{2}}\right)$ with

$$
\begin{aligned}
\left(x_{1}, x_{2}, \ldots, x_{n}, x_{n+1}, \ldots, x_{n^{2}-1}, x_{n^{2}}\right) & =\left(e_{11}, e_{12}, \ldots, e_{1 n}, e_{21}, \ldots, e_{n(n-1)}, e_{n n}\right) \\
\left(y_{1}, \ldots, y_{n}, \ldots, y_{n^{2}-1}, y_{n^{2}}\right) & =\left(e_{11}, \ldots, e_{n 2}, \ldots, e_{(n-1) n}, e_{n 1}\right)
\end{aligned}
$$

where the $e_{i j}$ are the standard matrix units, $y_{1}=e_{11}, y_{n^{2}}=e_{n 1}$, and $y_{2}, \ldots, y_{n^{2}-1}$ are the unique choices of matrix units such that the monomial with $\sigma=1$ is nonzero. So $c_{2 n^{2}}$ takes on the value $e_{11} \neq 0$. Based on this example, we introduce the following definition:

Definition 1.1. We will say that a multilinear polynomial $f\left(x_{1}, \ldots, x_{t}\right) \in F\{X\}$ is a polynomial test for an $F$-algebra $R$ if it is not a polynomial identity for $R$ but it is an identity for every proper $F$-subalgebra of $R$.

Thus, the Capelli polynomial of total degree $2 n^{2}$ is a polynomial test for $M_{n}(F)$. Moreover, central polynomials for $M_{n}(F)$ are polynomial tests for $M_{n}(F)$ (see [6]). In 2 , it is proved that the standard polynomial of degree $2 n-2$ is a polynomial test for the subalgebra of upper triangular matrices of $M_{n}(F)$. The double Capelli polynomials are

$$
\begin{aligned}
h_{2 t-1}\left(x_{1}, \ldots, x_{t}, y_{1}, \ldots, y_{t-1}\right) & \sum_{\sigma \in S_{t}, \tau \in S_{t-1}}(\operatorname{sg} \sigma \tau) x_{\sigma(1)} y_{\tau(1)} x_{\sigma(2)} y_{\tau(2)} \cdots x_{\sigma(t-1)} y_{\tau(t-1)} x_{\sigma(t)}
\end{aligned}
$$

and

$$
\begin{aligned}
h_{2 t}\left(x_{1}, \ldots, x_{t}, y_{1}, \ldots, y_{t}\right) & \\
& =\sum_{\sigma, \tau \in S_{t}}(\operatorname{sg} \sigma \tau) x_{\sigma(1)} y_{\tau(1)} x_{\sigma(2)} y_{\tau(2)} \cdots x_{\sigma(t-1)} y_{\tau(t-1)} x_{\sigma(t)} y_{\tau(t)} .
\end{aligned}
$$

Note that $h_{2 t-1}$ and $h_{2 t}$ are multilinear and alternate in the $x_{i}$ and also in the $y_{j}$.

Formanek pointed out that $h_{4 n-2}$ is not a polynomial identity for $M_{n}(F)$ and asked for the least integer $m$ such that $h_{m}$ is a polynomial identity for $M_{n}(F)$. Chang [3] has proved that the double Capelli polynomial $h_{2 t}$ is a consequence of the standard polynomial $s_{t}$. A different proof that $h_{4 n}=0$ is a polynomial identity for $M_{n}(F)$, that uses a variation of Rosset's proof of the Amitsur-Levitzki theorem 
[10], was given by Giambruno-Sehgal in [7]. An elegant one-line proof of Domokos is given in [4], Example 2.2, p. 917.

In [5], Domokos obtained a generalization of Chang's theorem. Since it is important in these notes, the precise statement of Domokos's theorem is included below.

Let $x_{1}, \ldots, x_{d}, y_{1}, \ldots, y_{m}$ be noncommuting variables over $F$, and let $w_{1}, \ldots, w_{u}$ be monomials in $y_{1}, \ldots, y_{m}$ such that $w_{1}, \ldots, w_{u}$ is a reordering of $y_{1}, \ldots, y_{m}$. For a subset $\Pi \subseteq S_{d}$ and a monomial partition $\left\{w_{1}, \ldots, w_{u}\right\}$ of the set of variables $Y$ we put

$$
\begin{aligned}
& f_{\Pi}\left(x_{1}, \ldots, x_{d}, y_{1}, \ldots, y_{m} \mid w_{1}, \ldots, w_{u}\right)= \\
& \sum(\operatorname{sg} \mu) x_{\pi(1)} \cdots x_{\pi\left(d_{1}\right)} w_{\rho(1)} x_{\pi\left(d_{1}+1\right)} \cdots x_{\pi\left(d_{1}+d_{2}\right)} w_{\rho(2)} \cdots \\
& \cdots w_{\rho(u)} x_{\pi\left(d_{1}+\cdots+d_{u}+1\right)} \cdots x_{\pi\left(d_{1}+\cdots+d_{u+1}\right)},
\end{aligned}
$$

where the summation runs over all $\pi \in \Pi, \rho \in S_{u}, d_{i} \geq 1$ for $i=1, \ldots, u+1$ such that $d_{1}+\cdots+d_{u+1}=d$ and $\operatorname{sg} \mu$ is \pm 1 according to the parity of the permutation of the "underlying" variables $x_{1}, \ldots, x_{d}, y_{1}, \ldots, y_{m}$ in the corresponding term.

Theorem $1.2([5])$. The polynomial $f_{S_{d}}\left(x_{1}, \ldots, x_{d}, y_{1}, \ldots, y_{m} \mid w_{1}, \ldots, w_{u}\right)$ is contained in the $T$-ideal generated by the standard polynomial $s_{d}$.

Corollary 1.3 ([5]). We have the strengthened version of the result of [3] and [7] we mentioned above:

$$
\sum_{\sigma \in S_{2 n}, \tau \in S_{2 n-1}}(\operatorname{sg} \sigma \tau) x_{\sigma(1)} y_{\tau(1)} \cdots y_{\tau(2 n-1)} x_{\sigma(2 n)}=0
$$

is a polynomial identity for $M_{n}(F)$; moreover, it is a consequence of the standard identity $s_{2 n}=0$.

To see that $h_{4 n-2}$ is not a polynomial identity for $M_{n}(F)$, consider the substitution (double staircase)

$$
\begin{aligned}
& x_{1}=e_{11}, y_{1}=e_{12}, x_{2}=e_{22}, y_{2}=e_{23}, \ldots, x_{n}=e_{n n}, \\
& \quad y_{n}=e_{n n}, x_{n+1}=e_{n(n-1)}, y_{n+1}=e_{(n-1)(n-1)}, \ldots, x_{2 n-1}=e_{21}, y_{2 n-1}=e_{11}
\end{aligned}
$$

where the $e_{i j}$ are the standard matrix units. The only nonzero monomials in $h_{4 n-2}\left(x_{i}, y_{i}\right)$ are the $2 n-1$ even cyclic permutations of $x_{1} y_{1} \ldots x_{2 n-1} y_{2 n-1}$, and they all have positive sign. Thus

$$
h_{4 n-2}\left(x_{1}, \ldots, x_{2 n-1}, y_{1}, \ldots, y_{2 n-1}\right)=2 I-e_{11} .
$$

We finish this section with two useful properties of the double Capelli polynomials.

Proposition 1.4. $\quad$ (a) $h_{q+r}$ is a linear combination, with coefficients being 1 or -1 of evaluations of $h_{q} h_{r}$.

(b) The polynomial $h_{t}$ is a consequence of the identity $h_{s}$ for any $t \geq s$.

Proof. To prove (a) we show an explicit formula, where for simplicity we consider the following statement: $h_{2(q+r)-2}$ is a linear combination with coefficients being 1 or -1 of evaluations of $h_{2 q-1} h_{2 r-1}$. Let $t=q+r-1$. We partition the set of permutations $S_{t}$ by defining the equivalence relation $\sigma_{1} \sim_{q} \sigma_{2}$ if the images of the interval $[1, q]$ under $\sigma_{1}$ and $\sigma_{2}$ are the same set. Similarly, we partition the set of 
permutations $S_{t}$ by defining the equivalence relation $\tau_{1} \sim_{r} \tau_{2}$ if the images of the interval $[1, q-1]$ under $\tau_{1}$ and $\tau_{2}$ are the same set. Then we have

$$
\begin{aligned}
& h_{2 t}\left(x_{1}, \ldots, x_{t}, y_{1}, \ldots, y_{t}\right)= \\
& \sum_{\substack{\sigma \in S_{t} / \sim_{q} \\
\bar{\tau} \in S_{t} / \sim_{r}}}(\operatorname{sg} \sigma \tau) h_{2 q-1}\left(x_{\sigma(1)}, \ldots, x_{\sigma(q)}, y_{\tau(1)}, \ldots, y_{\tau(q-1)}\right) \\
& \left.h_{2 r-1}\left(y_{\tau(1)}, \ldots, y_{\tau(t)}, x_{\sigma(q+1}\right), \ldots, x_{\sigma(t)}\right) .
\end{aligned}
$$

The assertion in (b) follows immediately from (a).

\section{A polynomial test for the fUll MAtrix AlgEBRA}

The main goal of this section is to prove that $h_{4 n-2}$ is a polynomial test for $M_{n}(F)$. Before proceeding to the proof of this theorem we need some preliminaries and notation (cf. [8]). Let $\ell, m$ be positive integers such that $\ell+m=n$, and set

$$
E_{(\ell, m)}(F)=\left[\begin{array}{cc}
M_{\ell}(F) & M_{\ell \times m}(F) \\
0 & M_{m}(F)
\end{array}\right]
$$

an $F$-subalgebra of $M_{n}(F)$.

(i) Associated to $E_{(\ell, m)}(F)$ are canonical $F$-algebra homomorphisms

$$
\pi_{\ell}: E_{(\ell, m)}(F) \rightarrow M_{\ell}(F) \quad \text { and } \quad \pi_{m}: E_{(\ell, m)}(F) \rightarrow M_{m}(F) .
$$

Furthermore, identify $M_{\ell}(F)$ and $M_{m}(F)$ with

$$
\left[\begin{array}{cc}
M_{\ell}(F) & 0 \\
0 & 0
\end{array}\right],\left[\begin{array}{cc}
0 & 0 \\
0 & M_{m}(F)
\end{array}\right]
$$

respectively.

(ii) Associated to a subalgebra $A$ of $E_{(\ell, m)}(F)$ are homomorphic image subalgebras $A_{\ell}$ and $A_{m}$ in $M_{\ell}(F)$ and $M_{m}(F)$ respectively.

(iii) Set

$$
T_{(\ell, m)}(F)=\left[\begin{array}{cc}
0 & M_{\ell \times m} \\
0 & 0
\end{array}\right],
$$

the Jacobson radical of $E_{(\ell, m)}(F)$.

(iv) Recall that every $F$-algebra automorphism $\tau$ of $M_{n}(F)$ is inner (i.e., there exists an invertible $Q$ in $M_{n}(F)$ such that $\tau(a)=Q a Q^{-1}$ for all $a \in$ $\left.M_{n}(F)\right)$. We will say that two $F$-subalgebras $A, A^{\prime}$ of $M_{n}(F)$ are equivalent provided there exists an automorphism $\tau$ of $M_{n}(F)$ such that $\tau(A)=A^{\prime}$.

Lemma 2.1. Let $A$ be a subalgebra of $E_{(\ell, m)}(F)$ such that $A_{\ell}$ satisfies $h_{q}$ and $A_{m}$ satisfies $h_{r}$. Then A satisfies $h_{(q+r)}$.

Proof. The hypothesis that $A_{\ell}$ satisfies $h_{q}$ implies that the evaluation of $h_{q}$ on $A$ consists of matrices of the form

$$
\left(\begin{array}{ll}
0 & * \\
0 & *
\end{array}\right)
$$

Similarly, the hypothesis that $A_{m}$ satisfies $h_{r}$ implies that the evaluation of $s_{r}$ on $A$ consists of matrices of the form

$$
\left(\begin{array}{ll}
* & * \\
0 & 0
\end{array}\right) .
$$


Thus $A$ satisfies $h_{q} h_{r}$. Since $h_{q+r}$ is a linear combination of evaluations of $h_{q} h_{r}$, $A$ satisfies $h_{q+r}$.

Theorem 2.2. $h_{4 n-2}$ is an identity for any proper subalgebra of $M_{n}(F)$.

Proof. Let $A$ be a proper subalgebra of $M_{n}(F)$. If $A$ is simple, then it is a finitedimensional central simple algebra over its center $k$. Let $K$ denote the algebraic closure of $k$; then $A \otimes_{k} K$ is a simple $K$-algebra in a natural way (cf. [11], $\S 1.8$ ), with $\operatorname{dim}_{K}\left(A \otimes_{k} K\right)=\operatorname{dim}_{k}(A)$. Also, $A \otimes_{k} K \cong M_{t}(K)$ for some $t \leq n$. Since $A$ is a proper subalgebra of $M_{n}(F)$, it follows that $t<n$. Hence, by the AmitsurLevitzki theorem, $A \otimes_{k} K$ satisfies $s_{2 n-2}$. Since $h_{4 n-5}$ lies in the $T$-ideal generated by $s_{2 n-2}$, we have that $h_{4 n-5}(A)=0$. If $A$ is not simple, it can be embedded as an $F$-algebra in $E_{(\ell, m)}(F)$ for some suitable positive integers $\ell$ and $m$ (with $\ell+m=n$ ). Since $h_{4 \ell-1}$ and $h_{4 m-1}$ are identities for $M_{\ell}(F)$ and $M_{m}(F)$ respectively, we apply Lemma 2.1 to obtain that $h_{4 n-2}$ is an identity for $A$.

\section{A polynomial test For $E_{(\ell, m)}$}

In this section we show that the double Capelli polynomial $h_{4 n-3}$ is a polynomial test for the subalgebra $E_{(\ell, m)}$ of $M_{n}(F)$ for any positive integers $\ell, m$ such that $\ell+m=n$.

Proposition 3.1. $h_{4 n-3}$ is an identity for every proper subalgebra of $E_{(\ell, m)}$.

Proof. We consider all possible proper subalgebras of $E_{(\ell, m)}(F)$. First let us consider a subalgebra $A$ of $E_{(\ell, m)}$ such that $A_{\ell}$ is a proper subalgebra of $M_{\ell}(F)$. Then $h_{4 \ell-2}$ is an identity for $A_{\ell}$ as established in Theorem 2.2, and $h_{4 m-1}$ is an identity for $M_{m}(F)$. Thus, by Lemma 2.1, $h_{4 n-3}$ is an identity for

$$
\left[\begin{array}{cc}
A_{\ell} & M_{\ell \times m}(F) \\
0 & M_{m}(F)
\end{array}\right],
$$

and consequently an identity for $A$. Similarly, $h_{4 n-3}$ is an identity for every subalgebra of $E_{(\ell, m)}$ such that $A_{m}$ is a proper subalgebra of $M_{m}(F)$. Clearly, $h_{4 n-4}$ is an identity for the semisimple case

$$
\left[\begin{array}{cc}
M_{\ell}(F) & 0 \\
0 & M_{m}(F)
\end{array}\right] .
$$

It only remains to consider the case when the projections $A \rightarrow A_{\ell}$ and $A \rightarrow A_{m}$ are equivalent representations of $A$, which means that given $A$ there is a fixed matrix $T$ such that $T A_{\ell} T^{-1}=A_{m}$. It easily follows that in this case $A$ is equivalent to the $F$-subalgebra of the form

$$
\left\{\left[\begin{array}{ll}
a & c \\
0 & a
\end{array}\right]: a, c \in M_{\ell}(F)\right\} .
$$

In [2], Proposition 2.5, it is proved that the standard polynomial $s_{2 \ell}$ is an identity for this algebra; hence, $h_{2 n-1}$ is an identity for $A$.

Remark 3.2. The polynomial $h_{4 n-3}$ is not an identity for $E_{(\ell, m)}$. For instance, if $n=3$ and $A=E_{(1,2)}$, we have

$$
h_{9}\left(e_{11}, e_{11}, e_{12}, e_{22}, e_{22}, e_{23}, e_{33}, e_{33}, e_{32}\right)=2 e_{12} \text {. }
$$


Remark 3.3. The above ideas can be generalized to prove that the double Capelli polynomial $h_{4 n-t-1}$ is a polynomial test for the block upper triangular matrix algebra

$$
\left(\begin{array}{ccccc}
M_{\ell_{1}}(F) & & & * & \\
& M_{\ell_{2}}(F) & & * & \\
& 0 & & & M_{\ell_{t}}(F)
\end{array}\right) \text {. }
$$

\section{ACKNOWLEDGMENTS}

I am indebted to Professor Edward Letzter for his help and guidance. I am also grateful to the referee for many helpful comments and suggestions that have substantially improved these notes.

\section{REFERENCES}

1. S. A. Amitsur and J. Levitzki, Minimal identities for algebras. Proc. Amer. Math. Soc. 1, (1950), 449-463. MR0036751 (12:155d)

2. Daniel Birmajer, On subalgebras of $n \times n$ matrices not satisfying identities of degree $2 n-2$. Submitted to Linear Algebra and its Applications (2003).

3. Qing Chang, Some consequences of the standard polynomial. Proc. Amer. Math. Soc. 104 (1988), no. 3, 707-710. MR0964846 (89i:16014)

4. M. Domokos, Eulerian Polynomial Identities and Algebras Satisfying a Standard Identity. Journal of Algebra 169 (1994), 913-928. MR1302125 (95k:16030)

5. M. Domokos, A generalization of a theorem of Chang, Communications in Algebra 23 (1995), 4333-4342. MR 1352536 (96k:16038)

6. Edward Formanek, Central polynomials for matrix rings. Journal of Algebra 23 (1972), 129132. MR0302689 (46:1833)

7. A. Giambruno and S. K. Sehgal, On a polynomial identity for $n \times n$ matrices. Journal of Algebra 126 (1989), no. 2, 451-453. MR1024999 (90j:16031)

8. Edward Letzter, Effective detection of nonsplit module extensions. E-print ArXiv http:// arxiv.org/math.RA/0206141 (2002).

9. Ju. P. Razmyslov, The Jacobson radical in PI-algebras, Algebra i Logika 13 (1974), 337-360; English transl., Algebra and Logic 13 (1974), 192-204. MR0419515 (54:7536)

10. Shmuel Rosset, A new proof of the Amitsur-Levitzki identity, Israel J. Math. 23 (1976), 187188. MR0401804 (53:5631)

11. L. H. Rowen, Polynomial identities in ring theory. Academic Press, New York-London, 1980. MR0576061 (82a:16021)

Department of Mathematics and Computer Science, Nazareth College, 4245 East Avenue, Rochester, New York 14618

E-mail address: abirmaj6@naz.edu 